\title{
The Effects of Trypsin Inhibitor on Insulin Secretion Using Rat Pancreas in an Organ Bath
}

\author{
ASUKA MORITA $^{1 *}$, MOTOSHI OUCHI ${ }^{1}$, KEITARO SATOH $^{2}$, SHUNSUKE KOBAYASHI $^{3}$, \\ MISAO TERADA ${ }^{4}$, HIDEFUMI WAKASHIN $^{5}$, HIROE KON $^{6}$, KEITARO HAYASHI $^{1}$, NAOHIKO ANZAI ${ }^{4,7}$, \\ AKIRA SHIMIZU $^{8}$, HITOSHI SUGIHARA ${ }^{3}$, KENZO OBA $^{9}$ and TOMOE FUJITA ${ }^{1}$ \\ ${ }^{1}$ Department of Pharmacology and Toxicology, Dokkyo Medical University School of Medicine, Tochigi, Japan; \\ ${ }^{2}$ Department of Pharmacology, Meikai University School of Dentistry, Saitama, Japan; \\ ${ }^{3}$ Department of Endocrinology, Diabetes and Metabolism, Graduate School of Medicine, \\ Nippon Medical School, Tokyo, Japan; \\ ${ }^{4}$ Dokkyo Medical University School of Medicine, Tochigi, Japan; \\ ${ }^{5}$ Department of Regulatory Physiology, Dokkyo Medical University School of Medicine, Tochigi, Japan; \\ ${ }^{6}$ Research Center for Laboratory Animals, Dokkyo Medical University, Tochigi, Japan; \\ ${ }^{7}$ Department of Pharmacology, Chiba University Graduate School of Medicine, Chiba, Japan; \\ ${ }^{8}$ Department of Analytic Human Pathology, Nippon Medical School, Tokyo, Japan; \\ ${ }^{9}$ Kawaguchi Sakura Clinic, Saitama, Japan
}

\begin{abstract}
Background/Aim: We developed an experimental method to reproduce insulin secretion from isolated rat pancreas preparations using an organ bath system. However, secretion of trypsin, another pancreatic enzyme, interferes with insulin production in such systems. We aimed to ascertain the minimum trypsin inhibitor (TI), dose for obtaining a sustained, stable rate of insulin secretion. Materials and Methods: The action of TI (1-10 $\mu \mathrm{g} / \mathrm{ml})$ on pancreatic preparations of male Wistar-Imamichi rats in organ bath experiments was assessed by measuring insulin, amylase, and trypsin activity. Results: The level of insulin outflow remained steady in the TI-treated samples, in contrast to that in the untreated control, where insulin secretion decreased over time. The level of amylase outflow did not change significantly. Trypsin activity was significantly lower in the TI-treated samples than in the control. Conclusion: Even low concentrations of TI can maintain insulin secretion by inhibiting trypsin activity in organ bath experiments.
\end{abstract}

This article is freely accessible online.

*These Authors contributed equally to this study.

Correspondence to: Motoshi Ouchi, MD, Ph.D., Department of Pharmacology and Toxicology, Dokkyo Medical University School of Medicine, 880 Kitakobayashi, Mibu, Shimotsuga, Tochigi 3210293, Japan. E-mail: ouchi@ dokkyomed.ac.jp

Key Words: Insulin, amylase, pancreas, trypsin inhibitor, organ bath.
Insulin is an important metabolic hormone and plays a crucial role in maintaining homeostasis. Insufficient insulin secretion results in diabetes mellitus, a disorder characterised by high blood glucose levels. Insulin therapy and various other glucose-lowering agents, including sodium/glucose cotransporter 2 inhibitors, glucagon-like peptide-1 (GLP-1) receptor agonists, and dipeptidyl peptidase 4 inhibitors (1), have been used for ameliorating this disorder. Furthermore, insulin-sensitising agents, which reduce insulin resistance, have also been used since many years. However, currently, there is no curative therapy for diabetes mellitus. Recently, research on oral insulin delivery has gained pace $(2,3)$. Nevertheless, protection of insulin from enzymatic degradation has not been fully elucidated and remains a challenge.

Lately, the organ bath technique with magnus tube has been used to investigate organ/tissue function in physiological conditions $(4,5)$. We have previously confirmed that the level of insulin outflow increases upon both glucagon-like peptide1 (GLP-1) (6) and glucose stimulation in this experimental system. Pancreatic organ bath preparations have been found to be sufficiently sensitive and reproducible as a method to assess the effects of novel therapeutics for diabetes mellitus on insulin secretion. However, in our previous two studies, we observed that the level of insulin outflow decreased over time in the control group without stimulation $(6,7)$. Thus, we presumed that adding a bioactive substance would create a situation where basal insulin secretion would be sustained. We suspected that the presence of pancreatic digestive enzymes reduced insulin secretion in organ baths employing 
pancreas preparations. In our earlier studies, we added soybean-derived trypsin inhibitor (TI) to the isolation buffer with collagenase for isolating rat pancreatic acini (8) and parotid gland acini (9), to prevent cell damage from trypsin and trypsin-like proteases. Hence, we focused on TI as a candidate bioactive substance and studied the effect of a moderately high concentration $(100 \mu \mathrm{g} / \mathrm{ml})$ of TI (7). Addition of TI to the experimental solution rescued the reduction in insulin secretion. Additionally, an enzyme marker of tissue damage in the TI group tended to be lower than that in the control group.

The influence of TI on insulin secretion and hence on glucose metabolism is not completely known. However, if trypsinogen gets accidentally converted to trypsin within the pancreas, likely due to excessive pancreatic exocrine stimulation, disturbance of pancreatic duct flow, or the reflux of bile and intestinal fluid into the pancreatic duct, autolysis of the pancreas occurs, inducing pancreatitis, which leads to dysregulated glucose metabolism (10). Thus, adding TI suppresses trypsin-associated pancreatitis, which may reverse the reduction in insulin secretion from the pancreatic preparation. In the present study, we focused on finding the minimum TI dose for obtaining sustained and stable insulin secretion. Thus, the aim of this study was to assess the effect and the pharmacological action of TI at low doses, compared to those used for earlier investigations on insulin secretion, using organ bath systems.

\section{Materials and Methods}

Animals. Male Wistar-Imamichi rats (10-13 weeks old) were purchased from the Institute for Animal Reproduction (Ibaraki, Japan). The rats were housed under a controlled temperature of $23 \pm 3^{\circ} \mathrm{C}$ and lighting from 7:00 to 19:00 h. Laboratory food CE-2 (CLEA Japan, Inc., Tokyo, Japan) and water were provided ad libitum. The experimental protocols were approved by the Institutional Animal Care and Use Committee, Dokkyo Medical University (Approval number 1117, 27 th December 2017), and the experiments were conducted in accordance with the Guidelines for Animals Experimentation of Dokkyo Medical University. All efforts were made to minimise the number of animals used and their suffering. For analysis of trypsin, 24 rats of similar age $(80.7 \pm 1.2$ days) were assigned to four groups ( $\mathrm{n}=6$ for each group) receiving $0,1,3$, and $10 \mu \mathrm{g} / \mathrm{ml} \mathrm{TI}$, respectively. All applicable international, national, and/or institutional guidelines for the care and use of animals were followed.

Organ bath experiment. The organ bath system was used as described previously (6). Briefly, rats were euthanised humanely by carbon dioxide, and blood was taken from the caudal vena cava. The whole pancreas was removed from rats and subsequently immersed in modified Tyrode's solution. Next, the right lobe located on the duodenal side was separated, wrapped in a nylon mesh as the pancreas preparation, and suspended in the organ bath setup. The preparation was equilibrated for $120 \mathrm{~min}$; the solution in the bath was replaced with fresh solution every $20 \mathrm{~min}$. TI powder
(FUJIFILM Wako Pure Chemical Corporation, Osaka, Japan) was mixed with the incubation medium. To assess the effects of different TI concentrations $(0,1,3$, and $10 \mu \mathrm{g} / \mathrm{ml})$ on insulin and amylase outflow, $2.3 \mathrm{ml}$ of the incubation medium was sampled at four time intervals, i.e., at $0,20,40$, and $60 \mathrm{~min}$. The collected solutions were poured into Protein LoBind tubes (Eppendorf, Hamburg, Germany) and stored at $-80^{\circ} \mathrm{C}$ for further analyses. The weight of each pancreas was measured at the end of the experiment.

Quantitative determination of insulin, amylase, and lipase outflow. Insulin and amylase were measured in this experimental system as described previously (6). The levels of insulin in the collected samples were determined by an Ultra Sensitive Rat Insulin ELISA Kit (Morinaga Institute of Biological Science, Inc., Kanazawa, Japan) according to the manufacturer's instructions. The concentration of insulin in the samples was calculated using a standard curve.

Quantitative determination of amylase and lipase in the collected solutions was performed using a Fuji Dri-Chem 3500V DryChemistry Analyzer along with Fuji Dri-Chem Slide AMYL-PIII and v-LIP-P (Fujifilm Corp., Tokyo, Japan), following a previous study where rat serum lipase was quantified (11). The samples were diluted 10-fold with phosphate-buffered saline (PBS) and warmed at $37^{\circ} \mathrm{C}$. A $10 \mu \mathrm{l}$ aliquot of solution was taken on the slide for analysis. All measurements have been presented as the actual measurement divided by the weight of the unwrapped pancreas preparation.

Trypsin activity assay. Trypsin activity was measured at baseline and end of the experiment using a colorimetric assay kit (Bio Vision, CA, USA). Samples $(100 \mu \mathrm{l})$ were taken in a 96-well plate, and 1 $\mu \mathrm{l}$ of $50 \times$ chymotrypsin inhibitor solution was added to each sample, followed by incubation for $10 \mathrm{~min}$ at room temperature. Subsequently, $50 \mu \mathrm{l}$ of reaction mix $(48 \mu \mathrm{l}$ assay buffer $+2 \mu \mathrm{l}$ trypsin substrate) was added to each well and incubated at approximately $15-20^{\circ} \mathrm{C}$, while being protected from light. Absorbance at $405 \mathrm{~nm}$ was measured at baseline and after $2 \mathrm{~h}$ of incubation. One unit of trypsin activity was defined as the amount of trypsin required for cleaving the substrate to yield $1 \mu \mathrm{mol}$ of p-nitroaniline (p-NA) per min. Trypsin activity was calculated using a standard curve (linear between 0.02 to $80 \mathrm{nmol} /$ well) according to the formula provided in the manufacturer's protocol.

Stereomicroscopy of pancreas. The excised pancreas preparations were cut and immersed in red mercurochrome dye (Kozakai Pharmaceutical Co., Tokyo, Japan). The red dye was diluted 6-fold (high concentration) and 30-fold (low concentration) with modified Tyrode's solution before immersion for $1 \mathrm{~h}$ and $3 \mathrm{~h}$ at both concentrations. The immersed pancreas was washed three times with PBS and stored at $-80^{\circ} \mathrm{C}$. The cross-section of pancreas was observed using an SZH10 stereomicroscope (Olympus Optical Co., Ltd., Tokyo, Japan) after cutting the frozen tissue samples.

Histopathology of pancreas. Frozen pancreas was fixed with $10 \%$ buffered formalin. Formalin-fixed tissues were embedded in paraffin blocks, and $4 \mu \mathrm{m}$ thick sections were prepared. Sections were deparaffinised and stained with haematoxylin and eosin (H\&E) following standard protocols. Images were obtained using a light microscope. The total area of degenerative cells was analysed using the WinROOF image processing software (MITANI Corporation, Tokyo, Japan). 

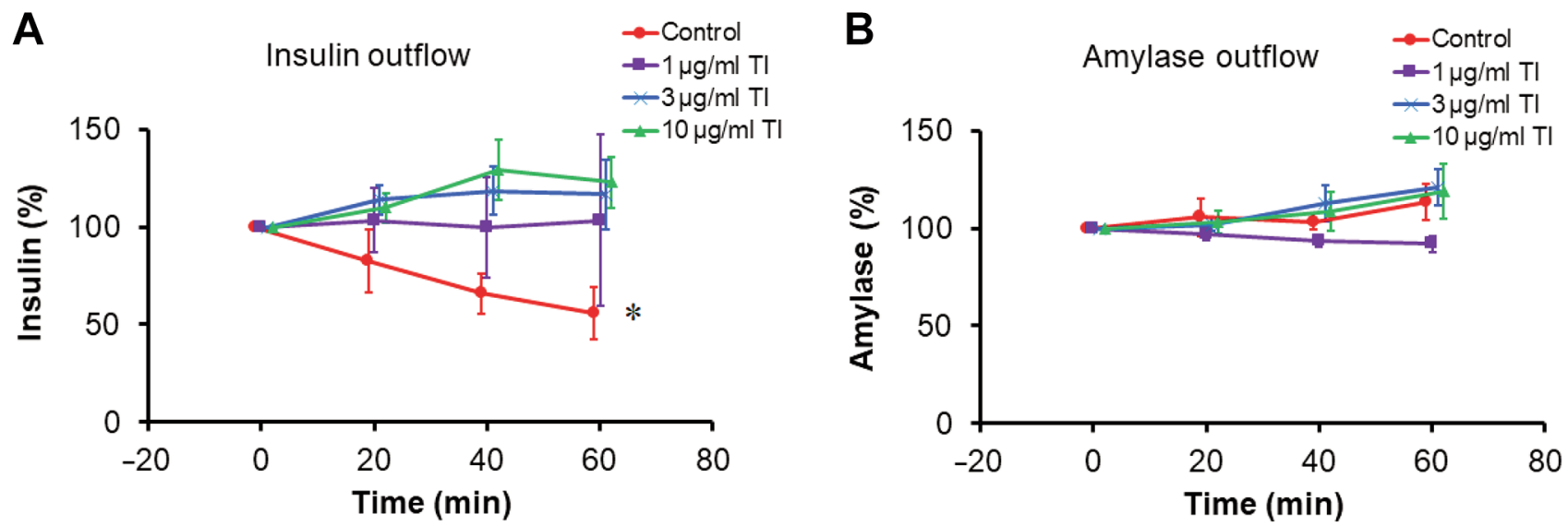

Figure 1. Effect of TI on secretory responses from rat pancreas preparations. (A) Insulin outflow. (B) Amylase outflow. Each point indicates data for tissues from six rats. Values represent the percentage of insulin and amylase outflow during 20-60 min incubation of samples in the TI-treated groups compared to that in the control and are shown as mean \pm S.E. To assess changes over time, repeated analyses of variance and the Bonferroni post hoc test were performed. ${ }^{*} p<0.05$. The level of insulin outflow in the control group decreased over time. TI: Soybean trypsin inhibitor.

Statistical analysis. Statistical tests were carried out using IBM SPSS ver. 25 (International Business Machines Corp., Armonk, NY, USA). To assess changes over time in the organ bath study, repeated measures analysis of variance and the Bonferroni post hoc test were performed. Significant differences in the experiments using organ baths were determined by one-way analysis of variance. Dunnett's post hoc test was used to detect significant differences between the control and treatment groups. Comparison of two groups was also performed using Student's $t$-test. Linear regression was used to estimate the insulin outflow achieved from trypsin activity and the lipase outflow achieved from amylase outflow. All values are expressed as means \pm standard error. $p$-values less than 0.05 were considered statistically significant.

\section{Results}

Effect of TI on insulin and amylase. We observed that TI affected insulin secretion. As shown in Figure 1A, the level of insulin outflow in the control group $(0 \mu \mathrm{g} / \mathrm{ml} \mathrm{TI})$ decreased over time ( $p=0.014$; baseline $v s$. $60 \mathrm{~min}, p=0.016$ ), whereas, the level of insulin outflow in the TI-treated groups did not vary significantly with time $(1 \mu \mathrm{g} / \mathrm{ml} \mathrm{TI}: p=0.948 ; 3$ $\mu \mathrm{g} / \mathrm{ml}$ TI: $p=0.345$; and $10 \mu \mathrm{g} / \mathrm{ml}$ TI: $p=0.135)$. Furthermore, the level of amylase outflow in the control and TI-treated groups did not change significantly over time (control: $p=0.326 ; 1 \mu \mathrm{g} / \mathrm{ml} \mathrm{TI}: p=0.082 ; 3 \mu \mathrm{g} / \mathrm{ml}$ TI: $p=0.084$; and 10 $\mu \mathrm{g} / \mathrm{ml}$ TI: $p=0.241$ ) (Figure 1B).

Trypsin activity in organ baths. Trypsin activity was significantly low in the TI-treated samples compared to that in the control [at baseline of experiment (Figure 2A): $p<0.001$; and at the end of experiment (Figure 2B): $p<0.001]$. Further, insulin outflow at the baseline was low when the trypsin activity was high $\left(\mathrm{R}^{2}=0.214, p=0.023\right.$; Figure $\left.2 \mathrm{C}\right)$.
Relation between amylase and lipase outflow. The relationship of lipase outflow was investigated as a base for amylase outflow. Amylase and lipase outflow were not significantly different between the control group and 10 $\mu \mathrm{g} / \mathrm{ml}$ TI group at baseline and the end of the experiment (Figure 3A-D). Linear regression revealed the predicted levels of lipase outflow in relation to amylase outflow at baseline $\left(\mathrm{R}^{2}=0.254, p=0.095\right.$; Figure $\left.3 \mathrm{E}\right)$ and at the end of the experiment $\left(\mathrm{R}^{2}=0.763, p<0.001\right.$; Figure $\left.3 \mathrm{~F}\right)$.

Dye permeation in pancreas. In pathology, red mercurochrome is often used as a marker to reveal the direction of tissue polarity (12). As shown in Figure 4, the pancreas was stained with red mercurochrome uniformly. Time-dependent changes in dye permeation were not prominent. However, gradual increase of dyeing intensity depending on concentration was visible. Red mercurochrome staining suggests that dye molecules can permeate the pancreas, thus indicating the likelihood of TI permeation into the pancreas.

$H \& E$ staining was carried out to acquire general pathological information on the pancreas preparations. As shown in Figure 5, adipose tissue degeneration (indicated via arrows) was slightly more pronounced in the control group than in the TItreated group. However, no significant difference in tissue structure was evident with or without TI, as indicated by digital image analysis $\left(n=3\right.$; control group: $22,584.1 \pm 5,924.8 \mu \mathrm{m}^{2}$; TI group: $\left.9,131.0 \pm 3,021.3 \mu \mathrm{m}^{2} ; p=0.113\right)$.

\section{Discussion}

In the present study, we investigated the effect of TI on insulin secretion experiments using rat pancreas preparations. 

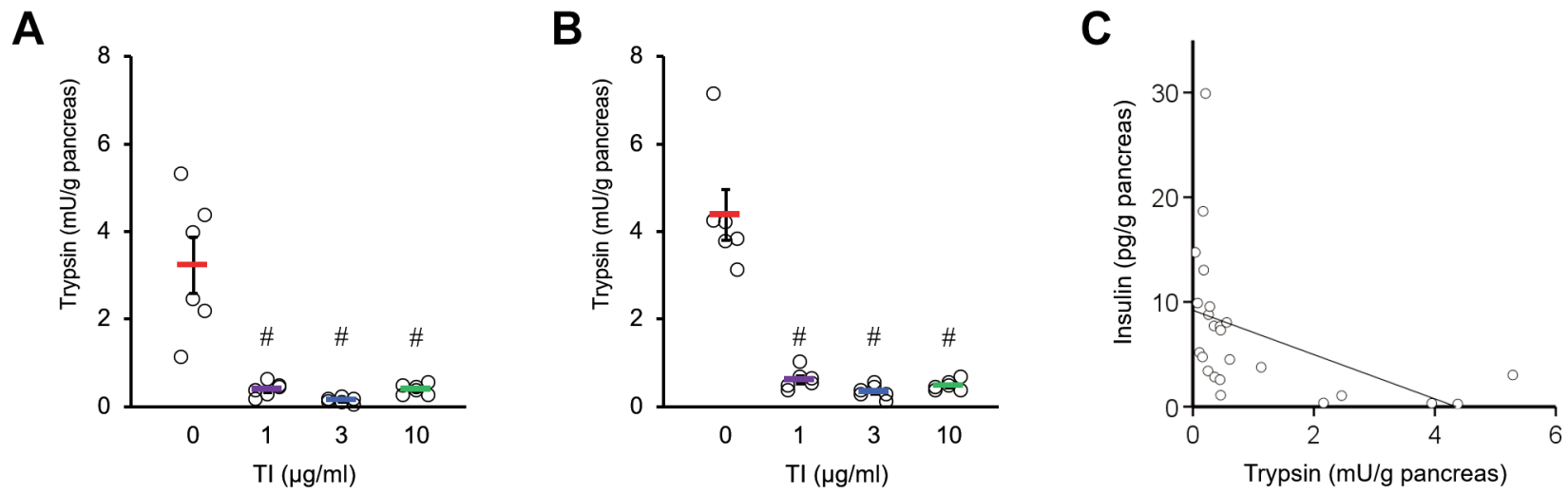

Figure 2. Effect of TI on trypsin activity. Trypsin activity at baseline $(A)$ and the end $(B)$ of the experiment. Data are expressed as mean $\pm S . E$. from six tissue samples. One-way analysis of variance and Dunnett's post hoc test were performed. ${ }^{~} p<0.001$ for TI-treated groups vs. control group. TI: soybean trypsin inhibitor. $(C)$ Insulin and trypsin activity baseline data at the beginning of the experiment. The linear regression equation for predicted levels of insulin outflow is as follows: predicted levels of insulin outflow=9.208-(2.114xtrypsin activity). Each open circle represents an individual value.

Emerging studies have indicated that insulin outflow decreases over time in such systems (6). The findings of our study indicate that TI has the potential to rescue the insufficient insulin outflow caused by trypsin.

The present study focused on assessing the effects of TI on insulin secretion using excised pancreas tissues in an organ bath. Banting and Best discovered insulin and observed its degradation by trypsin in the past $(13,14)$. Consistently, our results showed the rescue effects of TI on insulin in this experimental system. Since insulin is a peptide hormone, it is highly likely that it was eventually degraded by trypsin in samples from the pancreas preparations used in the present study. On the other hand, no destruction of the islets of Langerhans was observed in our study without TI as control, possibly because the observations were made prior to the onset of any structural disorder. However, trypsin could have disturbed the secretory function of $\beta$-cells in the pancreas preparations of the proposed system and thereby decreased insulin secretion. Further investigation using an in vitro model is required to confirm this possibility.

This pancreas preparation is partially removed from whole pancreas and trimmed in this experimental method. Earlier research has revealed high-level trypsin drainage after pancreatic anastomotic leakage (15) and higher trypsin levels were detected in exudates of selective drainage than in those of non-selective drainage after pancreatectomy (16). Thus, leakage of pancreatic juice containing trypsin is thought to occur due to selective drainage from remnant pancreatic stump. Further, serum insulin levels were reportedly reduced in patients with acute pancreatitis (10) and in a glucose- stimulated insulin secretion (GSIS) model of islets isolated from rats with acute pancreatitis (17). In another study, Toyota $e t a l$. reported that GSIS was inhibited by trypsin in isolated rat pancreas perfusion (18). However, limited investigation has been conducted on insulin secretion using pancreas preparations. In the present study, TI addition distinctly suppressed trypsin activity and maintained the ability of the pancreas preparation to secrete insulin. Therefore, it is plausible that trypsin activation is promoted by the transected pancreas.

Lactate dehydrogenase (LDH), an enzyme marker for tissue damage, is known to be released when the cell membrane breaks. LDH is fractionated as a cytoplasmic enzyme (19), which normally does not cross the cell membrane. LDH is also present in pancreas (20). We reported previously that there was a significant difference in results of LDH activity with $100 \mu \mathrm{g} / \mathrm{ml}$ TI addition, compared to control (7). In the present study, there was no significant difference in LDH activity (data not shown). Our findings suggest that minor injury-induced responses could have affected the results of this experiment. Due to the possibility of minor injury-induced responses in pancreas preparations, further investigation is required. Overall, it seems reasonable to conclude from the abovementioned different perspectives that insufficient insulin secretion is caused by pancreatitis and increased trypsin activity.

Our results propose that a concentration as low as $1 \mu \mathrm{g} / \mathrm{ml}$ of TI could be effective in suppressing trypsin activity. However, individual samples in the $1 \mu \mathrm{g} / \mathrm{ml}$ TI group had varying degrees of insulin. In our previous study, insulin outflow apparently increased slightly by $100 \mu \mathrm{g} / \mathrm{ml}$ of TI, a 
A

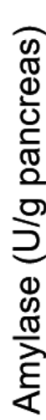

$\mathrm{Tl}(\mu \mathrm{g} / \mathrm{ml})$

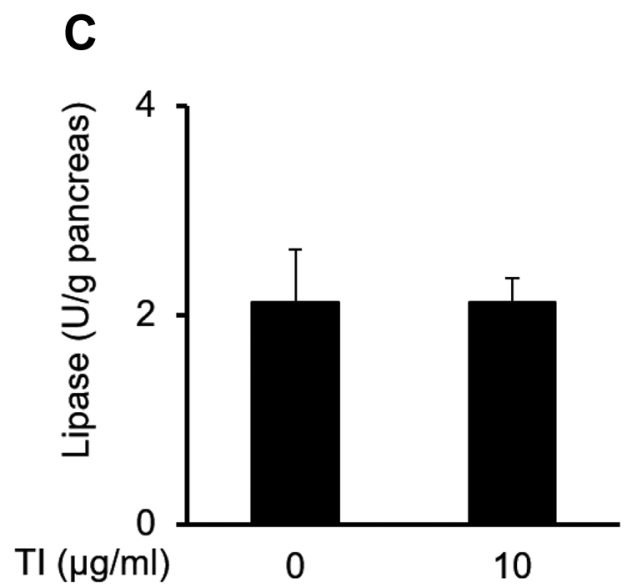

E

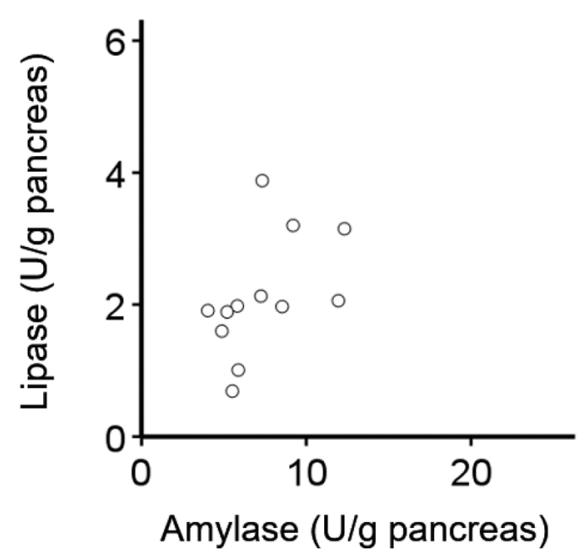

B

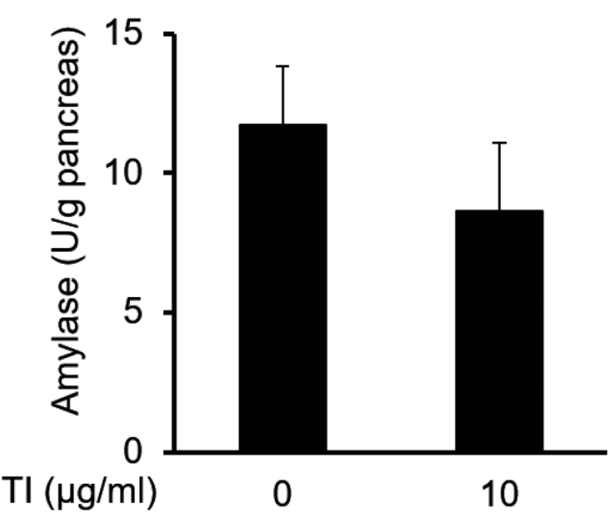

D

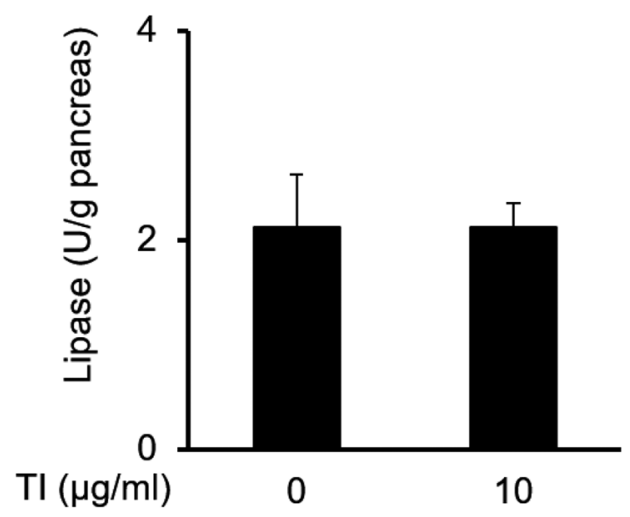

$\mathbf{F}$

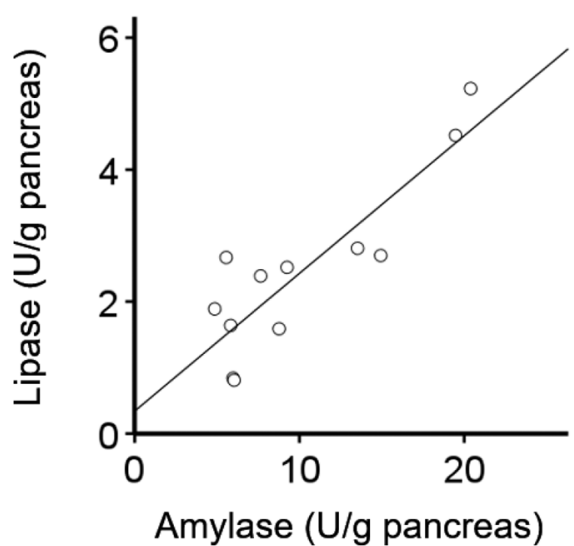

Figure 3. Effect of $10 \mu \mathrm{g} / \mathrm{ml} \mathrm{TI}$ on amylase and lipase outflow. (A, B) Amylase outflow. (C, D) Lipase outflow. Each bar indicates data for tissues from six rats. Data are expressed as mean \pm S.E from six tissue samples. Left bar is the control group and right bar is TI-treated groups. Left side $(A, C)$ is the data at baseline. Right side $(B, D)$ is the data at the end of the experiment. Comparison of two groups was also performed using Student's t-test [amylase: baseline (A): $p=0.107$; end of the experiment $(B): p=0.361$; lipase: baseline $(C)$ : $p=0.988$; end of the experiment $(D)$ : $p=0.985]$. Lipase and amylase outflow were plotted to examine the data at baseline $(E)$ and the end $(F)$ of the experiment. Each open circle represents an individual value. $(F)$ The linear regression equation for predicted levels of lipase outflow was as follows: predicted levels of lipase outflow $=0.341+(0.209 \times$ levels of amylase outflow $)$. TI: Soybean trypsin inhibitor. 


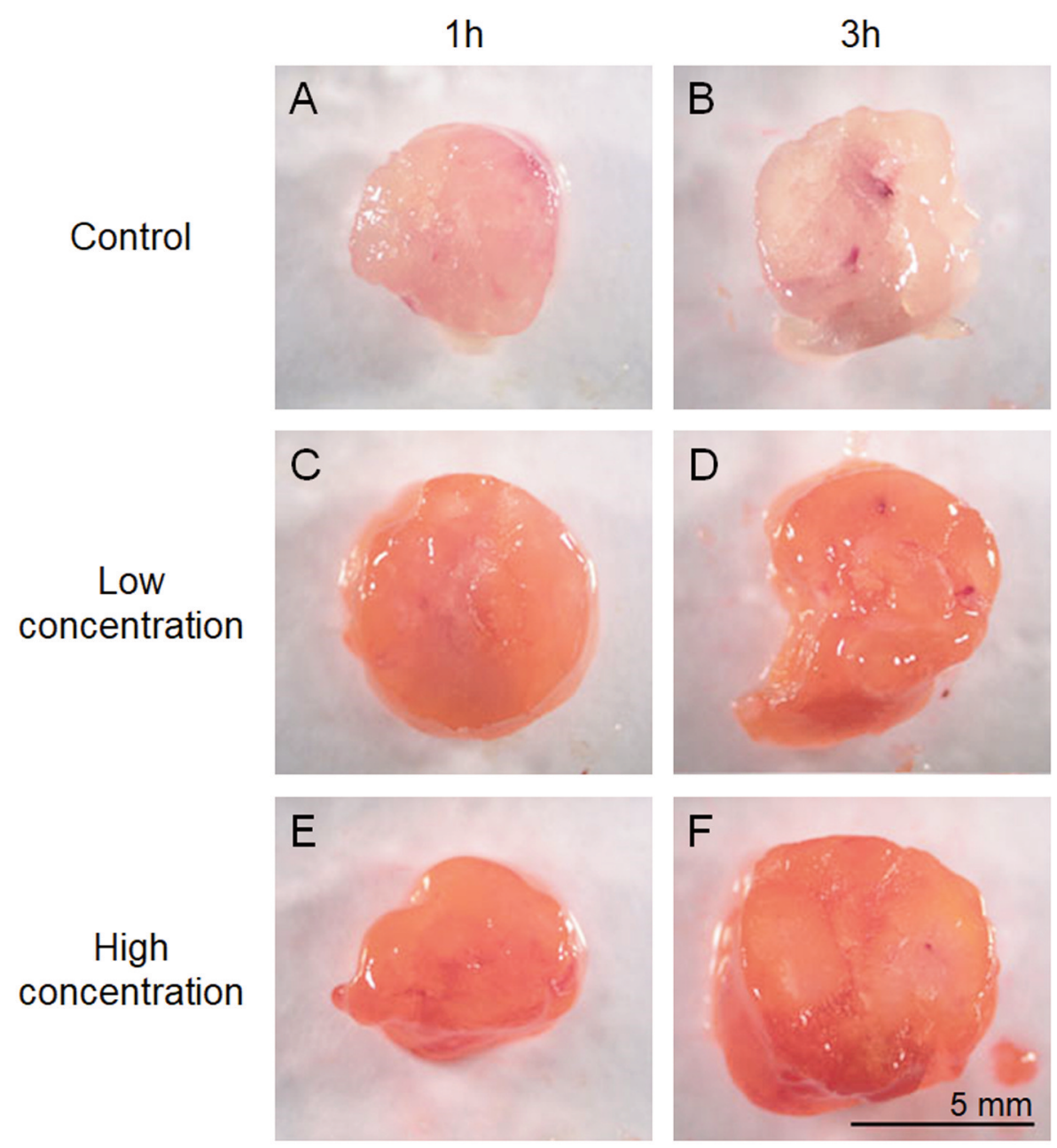

Figure 4. Stereomicroscopic features of the pancreas stained with red mercurochrome. The pancreas was incubated without red mercurochrome dye for $1 \mathrm{~h}(\mathrm{~A})$ and $3 \mathrm{~h}(\mathrm{~B})$. The pancreas was incubated with red dye diluted 30-fold (low concentration) for $1 \mathrm{~h}(C)$ and $3 h(D)$. The pancreas was incubated with red dye diluted 6-fold (high concentration) for $1 \mathrm{~h}(E)$ and $3 \mathrm{~h}(F)$. Scale bar: $5 \mathrm{~mm}$.

moderately high concentration. This concentration of TI may not only suppress trypsin activity, but may also have an enhancing effect on insulin secretion in such an experimental system. Thus, application of nearly $3 \mu \mathrm{g} / \mathrm{ml}$ TI to the organ bath is likely to be helpful in identifying substances for inducing insulin secretion in the future studies.

We identified the marked change that was recorded in insulin outflow, caused by TI treatment, was not observed in amylase outflow. We previously confirmed the variation in amylase outflow with and without $100 \mu \mathrm{g} / \mathrm{ml} \mathrm{TI}$ at the experimental end-point (data not shown) (7). Herein, we focused more on insulin outflow than on amylase and lipase outflow. Evaluation of the effect of TI on amylase and lipase levels by long-term protocols using this experimental system is likely to improve our understanding of the system.
In conclusion, the results of this study suggest that a small amount of TI reinforces the stability and effectiveness of this experimental system for studying insulin secretion. This outcome supports the utilisation of an organ bath technique for the evaluation of novel therapeutic approaches on insulin secretion. This system will also be useful to know the physiological activity and efficacy of other compounds.

\section{Funding}

This work was partly supported by a Grant from the Japan Society for the Promotion of Science (JSPS KAKENHI 18K15428, 19K10051, 20K11629).

\section{Conflicts of Interest}

The Authors declare that they have no conflicts of interest. 


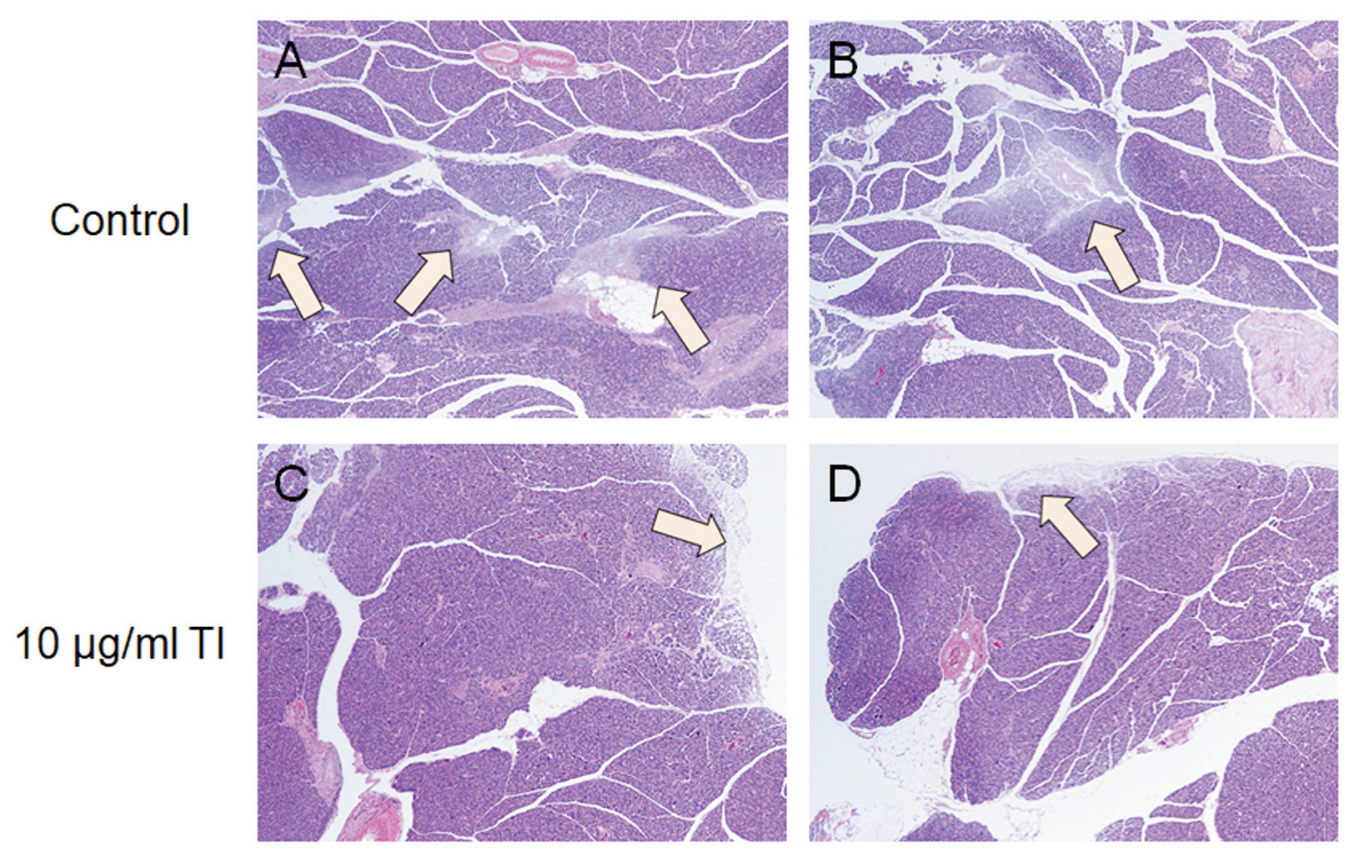

Figure 5. Haematoxylin-eosin $(H \& E)$ staining of the pancreas after the organ bath experiment. Representative H\&E stained specimens of control $(A, B)$ and $10 \mu \mathrm{g} / \mathrm{ml}$ TI-treated pancreas $(C, D)$. The degeneration of adipocyte (arrows) and acinar cells was observed. The total area of degenerative cells was measured in three selected acinar fields per pancreas using image analysis. There was no significant difference in the area with or without TI. Original magnification: $40 x$.

\section{Authors' Contributions}

MO and KO conceived the study. AM, MO, KS, MT, HW, HK, KH, $\mathrm{NA}$, and TF designed the experiments. AM, MO, MT, and HK contributed in developing the new methods. AM, MO, SK, MT, and AS performed the experiments. AM and MO analysed the experimental data. KS and HW contributed in data visualisation. AS and HS performed project administration. TF contributed in the acquisition of funding and resources. MO and KS prepared the initial draft of the manuscript. AM, MO, KS, SK, KH, NA, HS, $\mathrm{KO}$, and TF finalised the manuscript. All the Authors reviewed and approved the final manuscript.

\section{Acknowledgements}

The Authors are grateful to Dr. S. Kojima (Dokkyo Medical University School of Medicine) for his helpful and practical advice and Dr. K. Akimoto [No. 4 Common Laboratory (Cultures), Center for Research Collaboration and Support], F. Yokotsuka [No. 1 Common Laboratory (Morphological Science)], S. Nakadate, M. Maekawa (Dokkyo Medical University School of Medicine), and M. Kataoka (Nippon Medical School) for their expert technical assistance.

\section{References}

1 Tahrani AA, Barnett AH and Bailey CJ: Pharmacology and therapeutic implications of current drugs for type 2 diabetes mellitus. Nat Rev Endocrinol 12(10): 566-592, 2016. PMID: 27339889. DOI: $10.1038 /$ nrendo.2016.86
2 Niu M, Lu Y, Hovgaard L and Wu W: Liposomes containing glycocholate as potential oral insulin delivery systems: preparation, in vitro characterization, and improved protection against enzymatic degradation. Int J Nanomedicine 6: 11551166, 2011. PMID: 21822379. DOI: 10.2147/IJN.S19917

3 Ibie CO, Knott RM and Thompson CJ: Complexation of novel thiomers and insulin to protect against in vitro enzymatic degradation - towards oral insulin delivery. Drug Dev Ind Pharm 45(1): 67-75, 2019. PMID: 30252537. DOI: 10.1080/03639045.2018.1517776

4 Patejdl R, Gromann A, Bänsch D and Noack T: Effects of ajmaline on contraction patterns of isolated rat gastric antrum and portal vein smooth muscle strips and on neurogenic relaxations of gastric fundus. Pflugers Arch 471(7): 995-1005, 2019. PMID: 31044280. DOI: 10.1007/s00424-019-02279-y

5 Mumtaz FH, Lau DH, Siddiqui EJ, Thompson CS, Morgan RJ and Mikhailidis DP: Pharmacological properties of endothelin1 in the rabbit corpus cavernosum. In Vivo 20(2): 243-246, 2006. PMID: 16634525 .

6 Morita A, Ouchi M, Terada M, Kon H, Kishimoto S, Satoh K, Otani N, Hayashi K, Fujita T, Inoue KI and Anzai N: Reproducible insulin secretion from isolated rat pancreas preparations using an organ bath. Exp Anim 67(1): 15-22, 2018. PMID: 28757517. DOI: 10.1538/expanim.17-0059

7 Morita A, Ouchi M, Satoh K, Terada M, Kon H, Wakashin H, Hayashi K, Anzai N, Oba K, Shimizu A and Fujita T: Development of an organ bath technique for isolated rat pancreas preparations to assess the effect of 1,5-AG on insulin secretion. Exp Anim 69(2): 127-134, 2020. PMID: 31735767. DOI: 10.1538/expanim.19-0059 
8 Satoh K, Ouchi M, Morita A and Kashimata M: MARCKS phosphorylation and amylase release in GLP-1-stimulated acini isolated from rat pancreas. J Physiol Sci 69(1): 143-149, 2019. PMID: 29845509. DOI: 10.1007/s12576-018-0621-9

9 Satoh K, Seo Y, Matsuo S, Karabasil MR, Matsuki-Fukushima M, Nakahari T and Hosoi K: Roles of AQP5/AQP5-G103D in carbamylcholine-induced volume decrease and in reduction of the activation energy for water transport by rat parotid acinar cells. Pflugers Arch 464(4): 375-389, 2012. PMID: 22903161. DOI: $10.1007 / \mathrm{s} 00424-012-1141-8$

10 Drew SI, Joffe B, Vinik A, Seftel H and Singer F: The first 24 hours of acute pancreatitis. Changes in biochemical and endocrine homeostasis in patients with pancreatitis compared with those in control subjects undergoing stress for reasons other than pancreatitis. Am J Med 64(5): 795-803, 1978. PMID: 645743. DOI: 10.1016/0002-9343(78)90519-3

11 Nakamura T, Ichii O, Irie T, Kouguchi H, Sotozaki K, Chihara M, Sunden Y, Nagasaki KI, Tatsumi O, Elewa YHA and Kon Y: Cotton rat (Sigmodon hispidus) develops metabolic disorders associated with visceral adipose inflammation and fatty pancreas without obesity. Cell Tissue Res 375(2): 483-492, 2019. PMID: 30155650. DOI: 10.1007/s00441-018-2908-9

12 Shyamasundari K and Rao KH: A procedure for the simultaneous demonstration of neurosecretory and mucosubstances in tissue sections. Acta Histochem 54(2): 272-274, 1975. PMID: 56119.

13 Banting FG and Best $\mathrm{CH}$ : The internal secretion of the pancreas J Lab Clin Med 7(5): 251-266, 1922.

14 Scott DA: The action of trypsin on insulin. J Biol Chem 63: 641 651,1925

15 Kitamura A, Nomura E, Minami $\mathrm{N}$ and Kawai T: Clinical significances of simultaneous determination of trypsin and amylase activities in abdominal discharge after gastrointestinal surgery. Jpn J Gastroenterol Surg 24: 1230-1235, 1991 (in Japanese).
16 Arai K: [Studies on leakage of pancreatic juice following distal pancreatectomy from the standpoint of transition of amylase and trypsin values in exudate (in Japanese)]. Nihon Geka Gakkai Zasshi 95(2): 56-65, 1994. PMID: 7510016.

17 Kinami Y, Mura T, Sugii M and Miyazaki I: Function of pancreatic endocrine cells in experimental acute pancreatitis. World J Surg 6(4): 471-477, 1982. PMID: 6750930. DOI: 10.1007/BF01657685

18 Toyota T, Abe K, Kudo M and Goto Y: Effects of trypsin on glucose-induced insulin secretion from the perfused rat pancreas. Tohoku J Exp Med 118(4): 381-385, 1976. PMID: 779137. DOI: $10.1620 /$ tjem.118.381

19 Rasmussen HN, van Hall G and Rasmussen UF: Lactate dehydrogenase is not a mitochondrial enzyme in human and mouse vastus lateralis muscle. J Physiol 541(Pt 2): 575-580, 2002. PMID: 12042361. DOI: 10.1113/jphysiol.2002.019216

20 Wroblewski F and Gregory KF: Lactic dehydrogenase isozymes and their distribution in normal tissues and plasma and in disease states. Ann N Y Acad Sci 94: 912-932, 1961. PMID: 14008497. DOI: $10.1111 / \mathrm{j} .1749-6632.1961 . t b 35584 . x$
Received May 3, 2021

Revised May 31, 2021

Accepted June 1, 2021 